\title{
Espaces de l'eau : lieux féminins dans la littérature médiévale française
}

\author{
Bahíllo Sphonix-Rust, Emma
}

Universidad de Valladolid, sphemma@yahoo.es

\begin{abstract}
Resumen
Elemento femenino por excelencia, el agua aparece con frecuencia en la literatura medieval. Omnipresente en el ciclo artúrico, encontramos menos ejemplos en otros textos como es el caso de los cíclos épicos. Como tantos otros elementos, el agua integra la mentalidad medieval mediante diferentes valores, a menudo opuestos. Así, el agua dulce aparece en el espacio maravilloso céltico, mientras que en el paisaje épico se encuentra un agua particularmente destructora. A partir de un corpus heterogéneo de textos, se estudian elementos acuáticos diferentes como son el agua cristiana y el mar el Mediterráneo. Nuestro objetivo es mostrar cuáles son sus funciones -narrativas, simbólicas o poéticas- en el interior de los textos que conforman el corpus. Además se analizará cómo este elemento aparece asociado a la mujer.
\end{abstract}

Palabras clave : agua ; literatura ; Edad Media ; mujer ; espacio.

\section{Résumé}

Élément féminin par excellence, l'eau apparaît fréquemment dans la littérature médiévale. Omniprésente dans le cycle arthurien, elle se fait plus rare dans d'autres récits comme les cycles épiques. Comme bien des éléments, l'eau intègre la mentalité médiévale à travers des valeurs multiples, souvent opposées. Ainsi, l'eau douce est souvent liée à la féerie celtique, tandis que dans le paysage épique on retrouve une eau particulièrement destructrice. À partir d'un corpus hétéroclite de textes, nous étudions différents éléments aquatiques de nature différente comme l'eau chrétienne et la mer Méditerranée. Nous voudrions montrer quelles sont leurs fonctions -narrative, symbolique ou poétique- au cœur des textes. Nous nous demanderons également comment cet élément est associé à la femme, et notamment quelle est sa valeur féminine dans l'univers médiéval.

Mots-clés : eau ; littérature ; Moyen âge ; femme ; espace.

\begin{abstract}
Archetypal feminine element, the water appears frequently in the medieval literature. Omnipresent in the Arthurian cycle, she becomes more scarce in other narratives as the epic cycles. As many elements, the water integrates the medieval mentality through multiple, often brought into conflict values. So, the fresh water is often bound to the Celtic enchantment, whereas in the epic landscape we find a particularly destructive water. From a heterogeneous corpus of texts, we study various aquatic natural elements different as the Christian wate and, the Mediterranean Sea. We would want to show what are their functions -narrative, symbolic or poetic- in the heart of texts. We shall also wonder how this element is associated with the woman, in particular which is its feminine value in the medieval universe.
\end{abstract}

Keywords : Water ; literature ; Middle Ages ; woman ; space. 


\section{Introduction}

L'eau intègre la mentalité médiévale et, de ce fait, sa présence est attestée dans la littérature et notamment dans les récits du cycle arthurien. Elle apparaît moins souvent dans la tradition épique et surtout avec une autre signification : «l'eau épique est généralement plus naturelle, [...] elle est moins perfide, moins équivoque que l'eau celtique » (Régine Colliot, 1985). Dans la chanson de geste, l'eau est donc bien loin de cette eau douce souvent liée à la féerie celtique. En effet, dans le paysage épique se cache une eau particulièrement destructrice pour le chevalier. Et c'est que celui-ci est, en réalité, « héros terrien » (Colliot, 1985). Tel est le cas de Richard dans Fierabras ${ }^{1}$ qui, lorsqu'il s'apprête à (re)conquérir l'espace sarrasin, se heurte à cette eau périlleuse :

Par des[o]us cort une eve, Flagot est apele :

La rive a .XV. piez de haut tout mesure,

Et si descent plus tost que quarrel empene ;

Ne dromont ne galie n'i poient abite[r]. (v. 2582-2585)

Vers l'eve de Flagot a son resne tirre,

Venuz est a la rive soz un arbre rame,

Et voit l'eve bruiant, le flo parfont et le ;

Plus tost cort qu'ars ne trait un quarrel empenne ; (v. 4498-4500 ; 4502)

Richart esgarda l'eve, qui tant fait a douter ;

Tant fu grande et hydoisse qu'il n'i osa entrer.

Plus tost cort qu'ars ne trait, quant on en doit berser ;

Ne barge ne galie n'i poient habiter ;

Les rives en sont hautes, quanque on puet geter. (v. 4505-4509)

Cette fois, le danger aquatique se présente sous forme de fleuve : le Flagot. Sa description foisonne d'éléments qui soulignent son aspect redoutable. Sa taille est remarquable : «Cent toisses est parfonde, de lé liue et demie » (v. 4795); l'eau hydoisse- est extrêmement violente : son courant coule si vite que nul ne peut y naviguer. Du point de vue spatial, le Flagot intègre l'axe vertical par le biais de la profondeur, celle des eaux mais aussi celle des rives, qui tient lieu de muraille. A l'agressivité de l'espace se joignent les images guerrières comme l'évocation de la flèche. Le poème nous offre un visage négatif de l'eau. Cette image de l'eau s'accorde bien avec l'essence manichéenne de l'épique médiévale. En effet, l’univers médiéval est partagé entre deux valeurs morales clairement opposées : le Bien et le Mal.

Après avoir tracé une ébauche de l'eau épique et de sa fonction pour le chevalier, cette étude s'intéresse davantage à la question de l'eau et son rapport avec la figure féminine. Il s'agit de mettre en relief comment cet élément est associé à la femme et quelles sont ses valeurs dans un univers où la suprématie masculine est, comme l'a montré Georges Duby, un fait indéniable.

Dans ce contexte, notre analyse portera sur deux textes littéraires bien différents : Fierabras et La Fille du Comte de Ponthieu. Dans les lignes qui suivent nous en ferons une brève présentation dans l'ordre chronologique de production.

Composé vers 1170, Fierabras est une chanson de geste qui raconte la reconquête des reliques dérobées par les infidèles dans un texte antérieur : Destruction de Rome. Du point de vue thématique, la chanson possède une structure binaire. La première relate le duel entre Olivier et le méchant géant sarrasin Fierabras qui avait détruit Rome, tué le Pape et volé les reliques. Description et narration s'entremêlent dans la deuxième partie pour narrer les péripéties qui vont permettre aux chevaliers français de recouvrer leur bien.

Comme chacun sait, la chanson de geste loue les actions héroïques du chevalier. Il n’est pourtant pas seul. Souvent, une femme se cache à ses côtés. Dans Fierabras nous retrouvons une figure féminine surprenante : il s'agit de la fille de l'émir et de la sœur de Fierabras, Floripas. C'est dans la deuxième partie où la femme sarrasine va jouer un rôle essentiel. Car,

${ }^{1}$ Les citations de la chanson de geste renvoient à l'édition de Marc Le Person (2003) : Fierabras, chanson de geste du XII ${ }^{e}$ siècle. Paris : Champion (Les classiques français du Moyen Âge, 142). 
éprise d'amour pour un chevalier chrétien, elle va aider le champ ennemi à remporter la victoire et ainsi récupérer les reliques dérobées.

Le deuxième texte analysé s'éloigne du premier aussi bien du point de vue thématique que du point de vue de la forme. La Fille du Comte de Pontieu ${ }^{2}$ date du début du XIII ${ }^{\mathrm{e}}$ siècle. Dans son introduction de l'édition du texte, Clovis Brunel le classe comme une nouvelle :

on doit la considérer comme la plus ancienne nouvelle en prose française. Elle apparaît déjà avec le caractère très net de ce genre littéraire. Le récit rapide développe une action unique dont l’intérêt va se tendant jusqu’à un dénouement sous forme de trait (1929: III).

\section{L'eau bénite ou le pouvoir de transformation au féminin}

Floripas est une jeune fille sarrasine. Elle vit dans ce pays imaginaire qu'est l'Espagne épique. Elle s'inscrit donc juste à l’opposé de ce que représente une femme pour l’idéal chrétien. Elle est d'abord présentée par le biais d'un portrait qui surprend par sa longueur, et qui est considéré comme le seul vrai portrait du genre épique. La sarrasine possède une beauté extraordinaire : « Atant es Floripas, la fille a l’amirés ; /Ainz plus bele paienne ne vit nus quil soit nés. » (v. 2097-2098). L'auteur nous offre des indications qui concernent les formes -la bouche est petite, les lèvres « greilletes », le nez « bien sëant » et le front « droit et plané »- et les couleurs -la chair est « tendre et blanche », " la fache vermeillette », les dents " plus blanches d’yvoire reparré » et les lèvres «de roge i out plenté »-. Deux couleurs -fréquemment associées au Moyen Âge lorsqu'il s'agit de la beauté féminine- sont (re)utilisées dans ce portrait : le blanc et le rouge. La couleur blanche représente, comme l’affirme Jacques Ribard, «la pureté, la perfection » (1984 : 39). L’auteur participe du jeu ombre/lumière en situant son héroïne dans la lumière par le biais du blanc qui en est « la manifestation même » (1984 : 40). Outre la blancheur, un autre trait de l’héroïne vient renforcer cette lumière : la blondeur de ses cheveux.

Au cœur du portrait physique s’insère la description des vêtements (v. 2117-2138). Floripas est richement vêtue. Ce luxe vestimentaire s'exprime, comme l'affirme Jacques Le Goff, à travers « la qualité et la quantité de l'étoffe » (2008 : 330). Son costume est donc exceptionnel : la robe en soierie provenant de terres lointaines, l'or, utilisé dans chaque pièce, rappelle le luxe et renforce l'éclatante lumière du physique de Floripas. La jeune sarrasine incarne donc la femme séductrice, sa beauté et sa richesse la rendent désirable. Ainsi devient-elle, dans l'univers épique, une tentation pour le guerrier. Elle apparaît comme « l’un des leurres que la païennie présente au monde occidental » (Quéruel, 2000 : 205).

L’image de tentatrice exprimée par ce portrait se confirme avec son comportement, notamment lorsque Floripas fait des propositions indécentes aux chevaliers chrétiens emprisonnés sans les avoir encore vus.

En ce qui concerne le caractère, la princesse sarrasine est généralement imaginée comme un être coléreux et violent qui la distingue d'autres personnages féminins comme ceux des Romans de Tristan ou les Romans Antiques. Mais chez Floripas ce trait de caractère se présente de façon remarquable. Comme chacun sait, la colère est l'un des vices capitaux qui est, comme bien d'autres, associé à la femme. Chez elle ce comportement apparaît donc comme une forme de transgression qui doit être punie. Deux réactions suffiront pour l'exemplifier.

Peu de temps après son apparition, la sarrasine dévoile son caractère. Lorsqu'elle demande à voir les prisonniers français, Brutamont, geôlier de la prison, le lui interdit car « Souvent voit on granz maux par famë alever » (v. 2176); il renchérit en affirmant : « Maint preudonme ai veü par famë enganner » (v. 2183). Sa réaction face à ses paroles misogynes ne se fait pas attendre : sans le moindre doute elle tue le geôlier d'un coup de bâton et elle tient à le lui annoncer avant sur un ton ironique.

La païenne se montre une deuxième fois particulièrement violente, cette fois avec la parole, qui apparaît d'ailleurs comme la forme d'expression la plus courante de la colère féminine. Lorsque Sortibrant, conseiller de l'émir, dit qu'on ne peut faire confiance à Floripas de par sa nature, elle n’hésite pas à se servir d'insultes, en l'occurrence fiz a putains, pour se

\footnotetext{
${ }^{2}$ La Fille du comte de Ponthieu (1929). Éd. Clovis Brunel. Paris : Honoré Champion.
} 
défendre. Cette attitude n’est pas conforme à sa condition féminine, du moins pour système de valeurs de l'Occident chrétien.

À travers son comportement, Floripas incarne le monde sarrasin à qui l’imaginaire médiéval associe la violence : « [les païens] sont intimement et profondément violents » (2003 : 101), affirme Philippe Ménard. Mais la violence relève plutôt du monde masculin ce qui amène Paul Bancourt à parler de « la virilité des sarrasines épiques » (1982 : 640). Peut-être faut-il voir dans ce comportement un aspect séducteur qui vient compléter sa beauté physique et la rend encore plus désirable.

La fille de l'émir tombe follement amoureuse d'un chevalier chrétien, et elle va jusqu'à trahir son père pour gagner son amour. Tel qu'il a été décrit plus haut, Floripas ne correspond nullement au modèle d'épouse prôné dans l'univers chrétien. Comment va-t-elle imposer sa volonté ? Et c'est là que nous retrouvons l'eau, et plus précisément ce pouvoir se retrouve dans l'eau du baptême. En effet, c'est elle qui va permettre la métamorphose de la princesse sarrasine et, de ce fait, son triomphe.

Lors du baptême le trouvère nous dépeint une jeune fille qui ne ressemble nullement à la sarrasine présentée au début de la chanson:

\author{
La puciele despoullent, voiant tout le barné. \\ La car avoit plus blance que n'est flours en esté, \\ Petites mameletes, le cors grant et plané ; \\ Si cheveil resambloient fin or bien esmeré. (v. 6188-6191)
}

Quatre vers suffisent cette fois pour décrire la nouvelle image de la pucelle : son premier statut au regard de la religion chrétienne. Sous nos yeux s'efface cette apparence séduisante de la belle sarrasine. Le code vestimentaire y joue un rôle primordial puisqu'il apparaît comme le symbole de cette transformation. Floripas, en ôtant sa robe séductrice, se dépouille de son aspect luxueux pour revêtir une nouvelle identité, celle d’épouse chrétienne. Grâce au baptême, la princesse sarrasine est désormais une femme pure; sa nudité devient «l'habit de l'innocence, l'habit d'une Ève originelle » (Aramburu, 2001:15). Seul ce nouvel aspect lui permet d’intégrer une société si éloignée de la sienne, une société chrétienne où l'excès vestimentaire, le féminin notamment, est condamné avec virulence.

Micheline de Combarieu affirme à propos des sarrasines converties qu'il ne s'agit pas à proprement parler d'une conversion, mais plutôt d'une naissance (1979 :193). L'eau revêt ici une valeur positive car c'est cet élément qui rend possible la transformation de l'héroïne, son corps est désormais généré. La purification de la pécheresse à travers l’eau entraîne celle de tout l'espace sarrasin. Les noces peuvent maintenant se célébrer : «Tout droit enmi la plaice en sont avant alé ;/Iluec a l'arceveques l’un a l’autre douné. » (v. 6204-6205).

\title{
2. La mer Méditerranée : espace d'une renaissance
}

Le titre du deuxième texte que nous abordons ici -La fille du comte de Ponthieu- l'indique clairement: il s'agit de l'histoire d'une femme. Une femme, certes, mais sans existence autonome puisque, d'emblée, elle est présentée à travers sa relation avec l'univers masculin. Pour elle il n'existe qu'une seule destinée : se marier. Et c'est ainsi que s'ouvre le texte :

Li quens de Ponthiu, ki si estoit preudom, vit monseigneur Tiebaut de Domart, si l'apiela et le retint de sa maisnie, et quant il l'ot de sa maisnie, si en fu mout liés, car li quens monteplia en grant bien et en grant valour. Au repairier d'un tournoiement, apiela li quens monseignor Tiebaut, se li demanda et dist: “Tiebaut, lequel joel de ma tiere ameriés vous le miex? - Sire”, fait mesire Tiebaus, “jou sui uns povres hom, mais, se Diex m’aït, de tous les joiaus de vostre tiere jou n’ameroie nul tant comme madamoisiele vostre fille.”(1. 21-30). 
Quant à la structure du texte, il se divise en deux parties. Cinq ans après le mariage le couple n'arrive toujours pas à avoir de la descendance. Pour y remédier, ils décident de se rendre à Saint-Jacques-de-Compostelle. Comme Floripas, cette figure féminine ne s’identifie pas à l’image que la société médiévale attend de la femme. Contrairement à la princesse sarrasine nous ne connaissons ni son prénom ni son apparence physique. Toutefois, le texte nous offre des indices qui permettent de se l’imaginer. En effet, avant le départ elle insiste pour accompagner son époux en Galice puis finit par s'imposer. La femme devant rester dans un espace clôt qui l'enveloppe et la protège, l'idée de la jeune apparaît comme une forme de transgression. En dehors de l'espace privé toute sorte de dangers l'attendent : « "Dame, griés cose seroit a vostre cors, car la voie est mout lontaine et li païs mout estranges et mout diviers” » (1. 69-70).

Pour le Moyen-Âge la femme errante devient une femme impudique, incapable donc d'assurer la continuité du lignage. Peut-être cette décision est-elle le germe de ce qui advint par la suite. Lors du pèlerinage un malheureux événement vient rompre le bonheur conjugal : la jeune femme est violée par des brigands dans une forêt, lieu particulièrement dangereux pour elle. «Laide cose est a dame et a chevalier parmi forest passer a poi de compaignie » (l. 104-106), dit-on dans le texte. La scène du viol apparaît comme une rupture dans le récit et va engendrer des conséquences qui constituent la suite du récit. Victime de la violence des hommes, la femme réagit de la sorte à l'égard de son époux :

La dame va cele part u mesire Tiebaus giessoit et voit une espee jesir a tiere ki fu a uns des larons ki ochis fu, ele le prist et vait enviers son seignor plaine de grant ire et de mauvaise vollenté ki li estoit venue, car mout doutoit k'il ne l'en seust mal gret de çou ke il avoit veu et ke il ne li reprouvast en aucun tans et mesist devant çou c'avenu li estoit. Si dist : "Sire, jou vous deliverrai ja.” Lors haucha l'espee et vint viers son seignour et le cuida ferir parmi le cors. Il vit le cop venir, si le douta mout, car il estoit tous nus em pure sa chemise et ses braies sans plus, et si durement tressailli que les mains et li doit li furent deseure. Et ele le fiert si que ele le bleça et colpa les coroies de cui il estoit loiiés. Et quant il senti les loiiens laskier, il saca a lui, et rompi les coroies, et sailli sus em piés, et dist : "Dame, se Diu plaist, vous ne m’ocirés maishui!”. Et ele dist : “Certes, sire, çouu poise moi.” Il li toli l’espee et le remist el fuerre et apriés li mist la main sour l'espaulle et le remena a la voie k’il estoient venu (l. 158-175).

L'héroïne semble exprimer le sentiment douloureux que le viol fait naître en elle à travers cette tentative de meurtre de son mari. Mais ses actes ne sont pas sans conséquences puisque la jeune fille reçoit le pire des châtiments. Enfermée dans un tonneau, elle est jetée à la mer :

Li quens fist apareillier un fort batel et bien portant, si fist la dame entrer ens, et si i fist metre un touniel tout neuf, fort et grant, et fu pres. Il entrerent ens tout troi, sans plus de compaignie d'autre gent fors ke des marouniers ki les menerent, et se fist li quens nagier bien deus liues en mer, et mout s'esmierveilloit cascuns que il pensoit, mais nus ne li ossoit demander, Et quant il furent si avant en mer comme vous avés oï, li quens fist del tounel l'un des fons ferir hors, et prist la dame, ki estoit sa fille et ki mout estoit biele et bien acesmee, si le fist entrer el tonel, u ele vausist u non, et fist ferir le fons aprés li tantost et bien apareillier, et le pontenail fist tantost restouper et rajoindre ke eve n’i peust entrer en nule maniere. Li quens fist metre le touniel sor le bort de la nef. Il meismes ses cors empainst le touniel et bouta en la mer [...] (1. 265-279).

Cet extrait décrit dépeint la destinée de la jeune fille : il s’agit d’une véritable mise à mort.

La mentalité médiévale organise la réalité à partir d’oppositions. Chaque élément qui les compose possède un sens. Du point de vue spatial on retrouve par exemple l'axe vertical du haut et du bas, le premier ayant une valeur positive tandis que le deuxième est connoté négativement. C'est ainsi que nous retrouvons le Ciel et l'Enfer, chacun d'eux se rattachant à la lumière et aux ténèbres. Selon la pensée médiévale la mer apparaît comme une profondeur. L’élément aquatique est donc un lieu dangereux, un refuge de toute sorte d'êtres horribles : des monstres, des êtres hybrides comme un éléphant à queue de poisson ou encore un poisson à tête de chien et défenses de sanglier.

Cette vision de l'élément aquatique reçoit sans doute l'influence de la tradition biblique. À ce propos Alain Corbellari (2006) affirme : 
[...] les Hébreux sont un peuple du désert. La mer est pour eux un obstacle à éliminer [...] ou une tentation à éviter absolument : dans l'histoire de Jonas, la mer symbolise la lâcheté, la fuite, la désobéissance et, en fin de compte, la mort. C'est à peine si le Nouveau Testament est plus favorable à l'élément liquide : la mer de Galilée, qui n'est, de toute façon qu'un grand lac, est, de fait, aussi un espace indésirable ; on y calme la tempête et on y marche sur l'eau, comme pour mieux la nier.

La femme finit par survivre grâce à des marchands qui l'emmènent en Aumarie. Cet espace s’identifie probablement à la ville andalouse d'Almeria, mais dans le texte a l'apparence d'une île : «moi ne caut seur qel terrechou soit, mais ke jou soie hors de cest ille » (1. 537-539). Ce châtiment devient donc un véritable déplacement des côtes de la Manche jusqu’à la mer Méditerranée. Et la mer, cet espace aquatique, joue un rôle essentiel pour la jeune femme : elle apparaît comme le cadre d'une transformation voire d'une (re)naissance. « On plonge dans l'eau pour être rénové » affirme Gaston Bachelard (1942 : 197). De ces eaux surgit en effet une autre femme :

Il l'espousa quant ele fut renoie et criut en molt grant amour envers li, et petit fu avec lui quant elle conçut et eut un fil. Elle fu de le conpengnie a la gent et parla et entendi sarrasinois. Et petit demoura aprés qe ele eut une fille. Ensi fu bien deus ans et demi avoec le soudant, et entendi sarrasinois et parla molt bien (1. 280-286).

Ces quelques lignes suffisent pour témoigner de cette renaissance. Et justement on y utilise le mot « renoïe ». Elle renie sa foi chrétienne pour embrasser une autre religion fortement opposée à la sienne, et ainsi épouser le sultan -épris d'un amour fou pour cette jeune inconnue-. Elle maîtrise une nouvelle langue -le sarrasinois- puis elle donne naissance à un garçon et une fille. Une autre femme est maintenant née. Mais pour (re)naître il faut que son ancienne vie s'efface. Comment parvient-elle ? La jeune fille décide de se taire. Aux questions sur ses origines elle répond par le silence. Aux marchands qui la sauvent : «li marcheant li demanderent dint ele estoit, et ele leur cela verité » L. 324-325) ; au sultan qui la demande en mariage : « li fist requerre par latiniers k'ele li desist de quel gent ele estoit. Ele nule verité ne l'en valt dire ne counoistre » (1. 352-354). La transformation est telle qu'elle va jusqu'à dire : « Jou sui Sarrazine » (1. 542). Elle reconnaît également qu'elle possède des connaissances en magie : « [...] sai d'art et d'astrenomie» (1. 542-543).

Parmi tous ces éléments il en est un qui doit être remarqué. La genèse de la nouvelle, nous l'avons vu, est l'infertilité du couple mais en Aumarie la jeune femme réussit à donner la vie. L'eau joue de nouveau un rôle majeur puisque c'est cet élément qui va renverser la situation. Et c'est que « le contact avec l'eau comporte toujours une régénération : d'une part parce que la dissolution est suivie d'une "nouvelle naissance", d'autre part parce que l'immersion fertilise et multiplie le potentiel de vie » (Eliade, 1980 :199).

Après l'immersion dans la mer la jeune fille atteint la perfection de la condition féminine, c'est-à-dire remplir sa fonction productrice au cœur de la société. La mer devient donc le cadre d'une initiation.

Du côté de Ponthieu rien ne va plus. Depuis le châtiment de sa fille, la culpabilité accable le comte et monseigneur Thibaut n'ose plus se remarier et son frère ne veut plus devenir chevalier. Tous les trois décident donc d'entreprendre un nouveau pèlerinage en outremer. De retour, une tempête les surprend -« Quant il furent en haute mer, si les souprist uns vens durs et oribles, si que li maronnier ne seurent qel part il aloient» (1. 315-318)- mais les conduit en terre d'Aumarie. Ils ont échappé de peu à la mort, une mort qui les effraie comme affirme le comte : «de plus male mort ne de plus vilaine ne poons nous morir ke de morir en ceste mer » (l. 424-425). Il révèle en effet la peur chrétienne de mourir noyé car les corps engloutis par la mer ne peuvent ressusciter. La mer nous offre, certes, un visage dangereux. Mais ici le motif de la tempête possède en quelque sorte un aspect bienfaisant puisqu'il boulverse le destin des trois hommes : la mort était leur destin mais c'est elle qui les conduit vers des jours heureux.

Prisonniers du sultan, la jeune femme rompt son silence : « Sire, or poés vous bien dire ke vous iestes mes peres, et jou sui vostre fille et cele dont vous presistes si crueusse justiche, et vous, mesire Tiebaut, iestes me sires et mes barons, et vos, sire vallét, estes mes freres. » (l. 604-608).

Tous réussissent à quitter définitivement les terres sarrasines grâce à la ruse de la jeune femme. Rappelons ici d'ailleurs qu'il s'agit là d'un trait de caractère fréquent chez les sarrasines. Ensuite, elle regagne la Chrétienté en s'arrêtant à Rome : 
« Il reconcilia la dame et remist en droite crestientét, et confrema la dame et monseignour Tiebaut son baron en droit mariage et remist ensamble, et douna a cascun penitanche et les asolst de lor pechiés » (1. 723-726).

Il est vrai que les traversées sont mentionnés en très peu de lignes -peut-être est-il pour des raisons d'économie du récit. En réalité, les trajets maritimes sont des événements fondamentaux puisqu’ils structurent le récit. Ce sont les éléments qui vont renverser la diégèse pour arriver à cette fin heureuse. Ils apparaissent comme des va-et-vient entre deux pays et possèdent en outre une dimension symbolique qui valorise le pays d'accueil -Aumarie- par rapport au pays natal de l'heroïne -Ponthieu-. Dans ce récit la mer fonctionne comme un espace de disjonction ${ }^{3}$, c'est-à-dire elle oppose deux mondes en désignant un pays d'accueil et en stigmatisant une terre d'exil. Toutefois, cette condamnation du comté de Ponthieu disparaît à la fin du récit puisqu’il (re)devient terre d’accueil : « tantt ont erré k’il sunt venu el païs dont il sont né, et furent recheu a grant pourcession des evesques et des abés et des gens de relegion et l'autre clergie, ki mout les avoient desirés » (1. 730-733).

On reconnaît dans ce texte le rôle majeur de l'eau. De la mer surgit une femme autre, juste l'opposée de ce qu'elle était auparavant. Mais il s'agit là de la seule façon de survivre dans un espace qui n'est pas le sien, tel que l'avouera ellemême : « Jou sui Sarrazine renoïe, car autrement ne pooie durer, car jou fuisse piech’a morte » (l. 612-613).

Outre le fait de favoriser cette (re)naissance, l'eau contribue à ce que la fille du comte assume la fonction réservée aux femmes au cœur de la société : aussurer lascontinuité du lignage du comte de Ponthieu. D’autant plus que, rappelons-le, son infertilité est l’événenement déclencheur de la nouvelle.

\section{Conclusion}

Dans la littérature médiévale l'eau nous offre plusieurs visages. Dans la chanson de geste elle s’associe au paysage épique pour contribuer à l'aspect défensif des villes. Cet élément est également présent à travers la mer, étendue extraordinairement dangereuse qui est peuplée de toute sorte de bêtes fantastiques. Toutefois, l'eau dévoile une toute autre apparence lorsqu'elle se rapproche de la femme. Si dans un premier temps elle s'ébauche comme un espace de mort, elle en devient vite l'antithèse : espace salutaire qui lui permet de survivre tout en la métamorphosant. Une nouvelle femme surgit de ces eaux pour pouvoir enfin assumer le rôle que la société lui réserve, mais dans un territoire hostile au sien. Si l'eau est, certes, le cadre d'une initiation, elle apparaît également comme le milieu qui va permettre à la femme de (re)joindre -pour la fille de Ponthieu- ou d'atteindre -pour Floripas- l'idéal de perfection de la femme au Mouen Âge : « pia filia, morigera conjunx, domina clemens, utilis mater. Fille, épouse, dame et mère » (Duby, 1981:248).

\section{Références bibliographiques}

Aramburu Riera, Francisca (2001). «Le nu et le vêtu d’une princesse sarrasine dans Fierabras » dans Le nu et le vêtu, Senefiance, 47. Aix-en-Provence : Publications de l’Université de Provence, p. 7-16.

BAnCourt, Paul (1982). Les musulmans dans les chansons de geste du Cycle du Roi. Aix-en-Provence : Publications de l’Université de Provence.

Colliot, Régine (1985). « L’eau, élément du tragique : Textes du XIIIe siècle (geste de Doon de Mayence, Gui de Warewick) au XV siècle et documents iconographiques » dans L'eau au Moyen Âge [en ligne]. Aix-en-Provence : Presses universitaires de

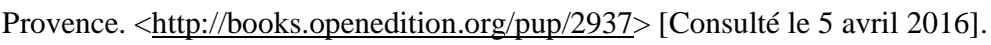

COMBARIEU Micheline de (1979). « Un personnage épique: la jeune musulmane » dans Mélanges de langue et littérature françaises du Moyen-Âge offerts à Pierre Jonin, Senefiance, 7. Aix-en-Provence : CUERMA.

Corbellari, Alain (2006). « La mer, espace structurant du roman courtois » dans Mondes marins du Moyen Âge [en ligne]. Aix-enProvence: Presses universitaires de Provence, <http://books.openedition.org/pup/3832> [Consulté le 9 avril 2016].

DuBY, Georges (1981). Le Chevalier, la Femme et le Prêtre. Le Mariage dans la France féodale. Paris : Hachette.

ELIADE, Mircea (1980). Images et symboles. Paris : Gallimard.

\footnotetext{
${ }^{3}$ Nous empruntons l'expression à Alain Cordellari.
} 
Fierabras, chanson de geste du XII siècle (2003). Éd. Marc Le Person. Paris : Champion (Les classiques français du Moyen Âge, 142).

La Fille du comte de Ponthieu (1929). Éd. Clovis Brunel. Paris : Honoré Champion.

LE GofF, Jacques (2008). La civilisation de l’Occident médiéval. Paris : Editions Flammarion.

Menard, Philippe (2003). «Le rire et les mentalités médiévales dans Fierabras : une réflexion sur la violence » dans Marc Le Person. Le rayonnement de Fierabras dans la littérature européenne. Actes du Colloque International des 6 et 7 décembre 2002. Lyon : Université Jean Moulin Lyon 3, Publication du CEDIC. p. 97-108.

Queruel, Danielle (1990). « L’histoire de la fille du comte de Ponthieu : distorsions et avatars d'une nouvelle » dans Alluin, Bernard et Suard, François. La Nouvelle : Définitions, Transformations. Lille : Université de Lille. p. 139-150.

Queruel, Danielle (2000). « Aimer et être aimé : le chemin de la conversion dans quelques textes du XIV et du XV siècle » dans $L a$ chrétienté au péril sarrasin. Senefiance, 46. Aix-en-Provence : Publications de l’Université de Provence. p. 203-218.

RiBARD, Jacques (1984). Le Moyen Âge. Littérature et symbolisme. Paris : Honoré Champion.

STANESCO, Michel (2005). « Mort et renaissance. La fille du comte de Ponthieu » dans Jacquart, Danielle ; James-Raoul ; Danièle et Soutet, Olivier. Par les mots et les textes... Mélanges de langue, de littérature et d'histoire des sciences médiévales offerts à Claude Thomasset. Paris : Presses de l’Université de Paris-Sorbonne (Travaux de stylistique et linguistique françaises. Série études linguistiques). p. 705-716.

SuARD, François (2012). « La fille du comte de Ponthieu: transgression, parole et silence » dans Monica L. Wright, Norris J. Lacy et Rupert T. Pickens. Moult a sans et vallour. Studies in Medieval French Literature in Honor of William W. Kibler, Amsterdam: Rodopi (Faux Titre, 378). p. 355-370. 